\title{
Determinación del coeficiente de estratificación horizontal y vertical de la ecuación modificada de Berlyand para fuentes fijas en la ciudad de Loja- Ecuador
}

Determination of the horizontal and vertical stratification coefficient of the modified Berlyand equation for fixed sources in the city of Loja-Ecuador

Thuesman Estuardo Montaño Peralta. ${ }^{1}$, Juan Carlos Solano Jiménez. ${ }^{2}$, Orlando Hilarión Álvarez Hernández. ${ }^{3}$, Carlos Andrés Mora Montaño. ${ }^{4}$, Wilson Cornelio Torres Ríos. ${ }^{5}$ \& Thuesman Humberto Montaño Ramón. ${ }^{6}$

\begin{abstract}
. https://doi.org/10.33262/concienciadigital.v4i1.2.1582

This research contributes to understanding the specific characteristics of the atmosphere in a locality to preserve and conserve people's health, ambient air quality, the well-being of ecosystems and the environment in general. Thus, the purpose of this research is to present the procedure and analysis carried out to obtain the horizontal and vertical stratification coefficient, and to determine the maximum concentration of pollutants from fixed point sources in the city of Loja - Ecuador, based on Berlyand's model. Through the research methodology, the predominant stability classes in the city of Loja were

\footnotetext{
${ }^{1}$ Facultad de la Energía, Universidad Nacional de Loja, Loja, Ecuador, thuesman.montano@unl.edu.ec

${ }^{2}$ Facultad de la Energía, Universidad Nacional de Loja, Loja, Ecuador, juan.solano@unl.edu.ec

${ }^{3}$ Consultor privado, Loja, Ecuador, orlando21alvarez@gmail.com

${ }^{4}$ Facultad de la Energía, Universidad Nacional de Loja, Loja, Ecuador, milton.leon@unl.edu.ec

${ }^{5}$ Facultad de Ciencias Agropecuarias, Universidad Técnica de Machala, Machala, Ecuador, wtorres@utmacha.edu.ec

${ }^{6}$ Ingeniería Mecánica, Universidad Politécnica Salesiana, Cuenca, Ecuador, thuesman92@gmail.com
} 
determined. Likewise, the average values of the dispersion parameters were determined. Finally, the value of the coefficient A of the Berlyand equation for the city of Loja was obtained analytically, whose value is 83 .

Keywords: Berlyand model, atmospheric stability, atmospheric stratification, solar radiation, wind speed.

\section{Resumen.}

Esta investigación contribuye al conocimiento de las características específicas de la atmósfera de una localidad con la finalidad de preservar y conservar la salud de las personas, la calidad del aire ambiente, el bienestar de los ecosistemas y del ambiente en general. Así, el propósito de esta investigación es presentar el procedimiento y análisis realizados para obtener el coeficiente de estratificación horizontal y vertical, y determinar la concentración máxima de contaminantes a partir de fuentes puntuales fijas en la Ciudad de Loja - Ecuador, a partir del modelo de Berlyand. Mediante la metodología investigación se logró determinar las clases de estabilidad predominantes en la Ciudad de Loja. Así mismo, se determinó los valores promedio de los parámetros de dispersión. Finalmente, se obtuvo analíticamente el valor del coeficiente $A$ para la Ciudad de Loja a partir de la ecuación de Berlyand, cuyo valor es de 83.

Palabras claves: Modelo de Berlyand, estabilidad atmosférica, estratificación atmosférica, radiación solar, velocidad del viento.

\section{Introducción.}

La contaminación del aire es uno de los grandes problemas que afecta a la mayoría de los países alrededor del mundo, especialmente a aquellos países industrializados y en vías de desarrollo. El incremento en las cantidades de gases contaminantes y de partículas potencialmente dañinas para la salud humana y el medio ambiente ha sido constatado a nivel mundial, y la respuesta a estos problemas se centra en la búsqueda de soluciones inteligentes (Delgado, M., et al, 2014), a corto, mediano y largo plazo, que detengan una contaminación del aire que podría ser irreversible en las próximas décadas.

Los logros obtenidos en la gestión de la calidad del aire contribuyen a la mejora del bienestar económico y social en muchos países en desarrollo (OMS, 2011). En este sentido, se ha comprobado que la gestión adecuada de la calidad del aire permite mejorar la salud pública, debido a que la contaminación atmosférica está relacionada con el aumento de pacientes ambulatorios, principalmente a causa de enfermedades respiratorias y cardiovasculares, y; por otro lado, al incremento de admisiones hospitalarias y de la mortalidad diaria.

La Ciudad de Loja se encuentra ubicada al Sur de la República del Ecuador, en el valle denominado de Cuxibamba, limitando con la cordillera occidental de los Andes. Loja 
tiene una superficie aproximada de $52 \mathrm{~km}^{2}$, con altitudes sobre el nivel del mar entre los 1950 y 2370 m (Fig. 1), y se encuentra entre las coordenadas siguientes: 03 ${ }^{\circ} 39^{\prime} 55^{\prime \prime}$ y $04^{\circ} 30^{\prime} 38^{\prime \prime}$ de latitud Sur (UTM 17 S: $9501249 \mathrm{~N}-9594638 \mathrm{~N}$ ); y, 790 05' 58" y $79^{\circ}$ 32' 42.1" de longitud Oeste (UTM 17 S: 661421 E — 711075 E).

Este territorio se caracteriza por gozar de un clima templado andino, a excepción de junio y julio, meses en los que se presenta una llovizna de tipo oriental (vientos alisos) con temperatura que fluctúa entre $\operatorname{los} 16^{\circ} \mathrm{C}$ y $25^{\circ} \mathrm{C}$. La época de mayor estiaje se presenta entre octubre y diciembre con una precipitación media anual que oscila entre 400 y 1100 mm (GEO, Loja. 2006).

La Ciudad de Loja tiene una población de 214855 habitantes y una tasa de crecimiento de $23 \%$, según lo establece el último censo realizado por el INEC (2010), lo que ha incidido en una clara expansión de viviendas y con ello la demanda de servicios que afectan el medio ambiente. Uno de los principales contaminantes que tiene la ciudad debido a la urbanización - radica en la explotación de fuentes fijas estacionarias, tales como los calefones, cuyo combustible o portador energético es el gas licuado de petróleo (GLP), el cual emana gases como CO2, $\mathrm{CO}, \mathrm{NOx}$, los cuales inciden negativamente en el medio ambiente por constituirse en gases de efecto invernadero (GEI). De igual manera, existen ocho fuentes fijas que expulsan a la atmósfera gases de efecto invernadero, especialmente $\mathrm{CO} 2$, NOx, y en menor medida $\mathrm{SO}_{2}$, cuyas ubicaciones se presentan también en la Fig. 1.

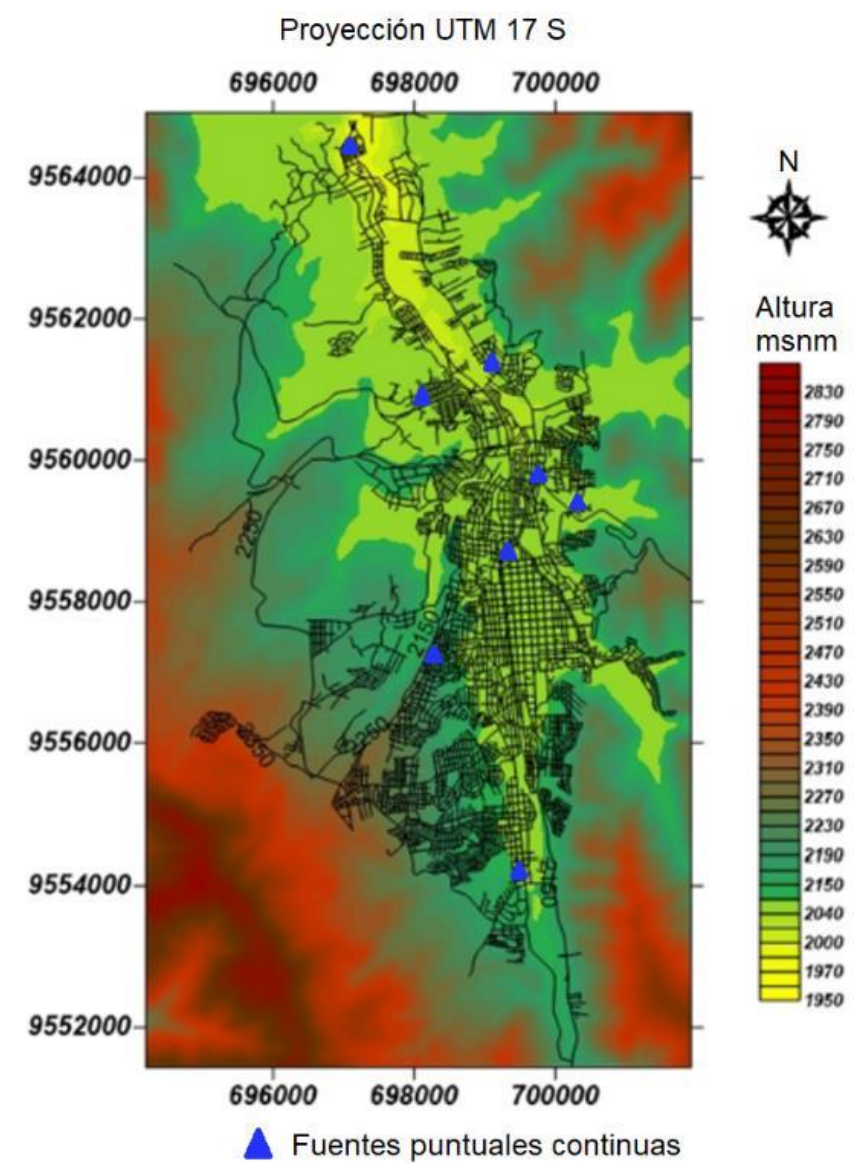


Figura 1. Mapa de la Ciudad de Loja y ubicación de las fuentes puntuales fijas.

En la Ciudad de Loja, además de la contaminación atmosférica generada por fuentes fijas, existe un crecimiento sostenido del parque automotor (CEPAL 2008). Por lo general, las emisiones de una sola unidad de cualquier vehículo son muy bajas comparadas con las emisiones de una chimenea industrial, sin embargo, debido a la gran cantidad de vehículos automotores en circulación, representan la fuente principal de contaminación que, según datos oficiales, en 2017, Loja contaba con aproximadamente 36000 vehículos en circulación (Agencia Nacional de Tránsito, 2014).

Aunque la evaluación completa de ubicación de fuentes grandes y específicas de contaminación requiere a menudo información detallada obtenida en el sitio, la información climatológica para las localidades cercanas puede ser útil en la planificación preliminar (Holzworth, 1974). Por lo tanto, los datos climáticos son indispensables en la evaluación de medidas de calidad del aire relacionada a las prácticas de control de emisiones y tendencias de calidad del aire. Durante los días de la semana (es decir los días de trabajo regulares), cuando las proporciones generales de emisiones de contaminantes en una ciudad pueden variar poco de día a día, las variaciones observadas en las concentraciones del contaminante son causadas por las variaciones en los rasgos de tiempo pertinentes (Holzworth, 1974). Por otro lado, el ciclo diario del calentamiento y enfriamiento del suelo bajo la acción de la radiación del sol, así como la mezcla de masas de aire de procedencia diferente, tiene como consecuencia la modificación del valor de la temperatura del aire en función de la altura. Esta modificación repercute en la habilidad de la atmósfera en iniciar o inhibir los movimientos verticales del aire (Neiburger, 1969). De esta manera, los datos disponibles requieren un procesamiento especial e interpretación en lo que se refiere a su impacto en el transporte atmosférico y difusión.

Los datos climatológicos en ocasiones resultan difíciles de interpretar debido a dos razones principales. En primer lugar, las observaciones no son en absoluto hechas en todos los lugares para los cuales se requiere la información, ni en las suficientes locaciones para permitir una interpolación fácil. Por ejemplo, cuando la preocupación es acerca de la contaminación atmosférica en una ciudad y las observaciones han estado hechas en un aeropuerto cercano, estos datos deben interpretarse en términos de los efectos que la ciudad tendría sobre las observaciones (Neiburger, 1969). Pero, incluso en el caso donde un sitio de observación está dentro de una ciudad, esas observaciones no pueden ser representativas de todas las secciones de la ciudad. Para una fuente puntual específica de contaminación es deseable tener las observaciones en la vecindad inmediata de la fuente. La segunda razón, se debe a que las observaciones, sobre todo del aire superior, no son hechas con la frecuencia suficiente. El transporte y características de la difusión de la atmósfera cerca de la tierra (en algunos lugares hasta varios kilómetros) normalmente exhiben una variación diurna muy grande, que es difícil de interpretar en ausencia de observaciones. 
Si bien existen modelos de dispersión de gases contaminantes recomendados por la USEPA (U.S. Environmental Protection Agency), no es menos cierto que los mismos trabajan en función de los datos meteorológicos existentes de cada localidad y los datos meteorológicos a utilizarse, deberán ser representativos para la ubicación geográfica de la fuente fija a evaluarse. En la Ciudad de Loja no existen estudios de aire superior, solamente en tres zonas orográficas cuyas condiciones son diferentes a Loja se ha realizado estudios aerológicos (Montaño, T., 2015).

\section{Metodología.}

Se utilizó el software Excel de Microsoft Office para realizar los procesamientos de las diferentes variables meteorológicas, así como herramientas CAD (Computer Aided Design), específicamente el software Surfer ${ }^{\circledR}$ para utilizar los mapas de la Ciudad de Loja y geolocalizar las fuentes fijas. Los datos de altitud se interpolaron a partir de los datos del Shuttle Radar Topographic Model (SRTM) de la NASA (National Aeronautics and Space Administration).

Las variables meteorológicas utilizadas normalmente en los estudios de contaminación atmosférica comprenden la dirección y la velocidad del viento, la temperatura ambiente, la cantidad de cielo cubierto por nubes, la altura de la base de las nubes, humedad y presión, los cuales son considerados datos meteorológicos primarios. Por otra parte, los datos secundarios y la forma en la cual son identificados se muestran en la Tabla 1.

Tabla 1. Parámetros meteorológicos secundarios para estudios de contaminación atmosférica

\begin{tabular}{lc}
\hline \multicolumn{1}{c}{ Parámetros } & Identificación \\
\hline Categorías de estabilidad atmosférica & $A, B, C, D, E, F$ \\
Altura de la capa de mezcla urbana y rural & $h$ \\
Exponente de perfil de viento & $p$ \\
Gradiente vertical y gradiente potencial vertical & $\Delta T / \Delta \mathrm{Z}$ \\
Longitud de Monin-Obukhov & $L$ \\
Velocidad de fricción & $U$ \\
\hline
\end{tabular}

Fuente: Turtós y otros, 2004.

En el presente trabajo, se utilizó el modelo de difusión turbulenta de la Teoría de Transporte Gradiente $K$, que se utiliza como modelo de control, para determinar zonas con posibles valores elevados de concentración de contaminantes gaseosos. A pesar de que este parámetro requiere de una integración numérica que es computacionalmente de difícil acceso, se puede resolver analíticamente con algunas simplificaciones. A partir de este modelo, se pueden hacer estimados confiables ( $25 \%$ - 30\% de error) de la dispersión de impurezas sin necesidad de estudios detallados de las construcciones urbanas (Berlyand, 1975). Además, se puede considerar el más simple y, presumiblemente el más crudo tipo de aproximación utilizado en la modelación de la difusión (Arya S Pal, 2002). 
Debido a lo anterior, y utilizando una estación meteorológica —instalada a 10 metros de altura en la Facultad de la Energía de la Universidad Nacional de Loja (UNL), la cual cuentan con sensores de radiación solar global, dirección y velocidad del viento, y precipitación - se adaptó el método de Pasquill-Turner (Turtós y otros, 2004;Moragues, J.A., 2015), como se muestra en la Tabla 2, para estimar la estabilidad vertical de la atmósfera, obteniéndose esta información desde marzo de 2013 hasta 2015. Además, estos datos están siendo obtenidos en tiempo real desde agosto de 2020 hasta la actualidad. En un trabajo de investigación sobre estabilidad vertical de la atmósfera en la provincia de Loja (Álvarez, Maldonado y Montaño, 2015), se presentaron los resultados preliminares obtenidos a partir de la información meteorológica de cinco estaciones automáticas administradas por la UNL, entre ellas, la ubicada en la ciudad de Loja.

Tabla 2. Turbulencia de la atmósfera y clases de estabilidad.

\begin{tabular}{|c|c|c|c|c|c|}
\hline \multirow{2}{*}{$\begin{array}{c}\text { Velocidad } \\
\text { del viento a } \\
10 \mathrm{~m} \text { de } \\
\text { altura } \\
(\mathrm{m} / \mathrm{s})\end{array}$} & \multicolumn{3}{|c|}{$\begin{array}{c}\text { Día (1) } \\
\text { Radiación solar incidente }\end{array}$} & \multicolumn{2}{|c|}{ Noche (2) } \\
\hline & $\begin{array}{c}\text { Fuerte } \\
>\mathbf{5 0 ~} \mathrm{cal} / \mathrm{cm}^{2}\end{array}$ & $\begin{array}{c}\text { Moderada } \\
25-50\end{array}$ & $\begin{array}{l}\text { Débil } \\
<25\end{array}$ & $\begin{array}{c}\text { (3) } \\
\mathbf{4 / 8}<\text { nubosidad }<7 / 8\end{array}$ & nubosidad $<\mathbf{3 / 8}$ \\
\hline$<2$ & A & $A-B$ & B & $\mathrm{F}$ & $\mathrm{F}$ \\
\hline $2-3$ & $A-B$ & B & $\mathrm{C}$ & E & $\mathrm{F}$ \\
\hline $3-5$ & B & $B-C$ & $\mathrm{C}$ & $\mathrm{D}$ & E \\
\hline $5-6$ & $\mathrm{C}$ & $C-D$ & $\mathrm{D}$ & $\mathrm{D}$ & $\mathrm{D}$ \\
\hline$>6$ & $\mathrm{C}$ & $\mathrm{D}$ & $\mathrm{D}$ & $\mathrm{D}$ & $\mathrm{D}$ \\
\hline $\begin{array}{l}\text { (1) Las unidades } \\
\text { (2) La noche se d } \\
\text { (3) La nubosidad } \\
\text { 0/8: Cielo des } \\
\text { 4/8: La mitad } \\
\text { 8/8: Cielo tot }\end{array}$ & $\begin{array}{l}\text { radiación solar s s } \\
\text { ne como el perío } \\
\text { mide en octavos } \\
\text { jado } \\
\text { l cielo cubierto } \\
\text { nente cubierto. }\end{array}$ & $\begin{array}{l}\text { xpresan en cal } \\
\text { desde una hor } \\
\text { cual significa }\end{array}$ & $\begin{array}{l}\text { en } \mathrm{kWl} \\
\text { lés de l} \\
\text { iiente: }\end{array}$ & $\begin{array}{l}\text { onde } 1 \mathrm{cal} / \mathrm{cm}^{2}=0,01 \\
\text { a del sol, hasta una ho }\end{array}$ & $\begin{array}{l}6 \mathrm{kWh} / \mathrm{m}^{2} \\
\text { antes de la salida. }\end{array}$ \\
\hline
\end{tabular}

Al no contar con datos medidos ni observados de total de cielo cubierto con nubes durante la noche, se consideró que el cielo estaba parcialmente nublado cuando los valores de radiación global en horarios nocturnos eran igual a cero, mientras que si se obtenía algún valor superior a cero se tomó como con nubosidad igual o superior a 4/8 de cielo cubierto, considerando esos valores bajos de radiación difusa proveniente de las nubes (Álvarez, Maldonado y Montaño, 2015). En la Fig. 2 se muestra el porcentaje de casos por clase de estabilidad para la estación meteorológica de la UNL.

Además, a finales del año 2014, se instaló una estación meteorológica de dos niveles de altura $(10 \mathrm{~m}$ y $30 \mathrm{~m}$ ) para la medición de temperatura del aire y dirección y velocidad del viento, a partir de la cual se determinó los gradientes de estas variables. Con esta información, se utilizó un segundo método de comparación, utilizando el método del gradiente de temperaturas modificado (Turtós y otros, 2004), como se muestra en la Tabla 3a, debido a que la obtención del gradiente original se encuentra a 20 m y $120 \mathrm{~m}$ de altura (Tabla 3b). 


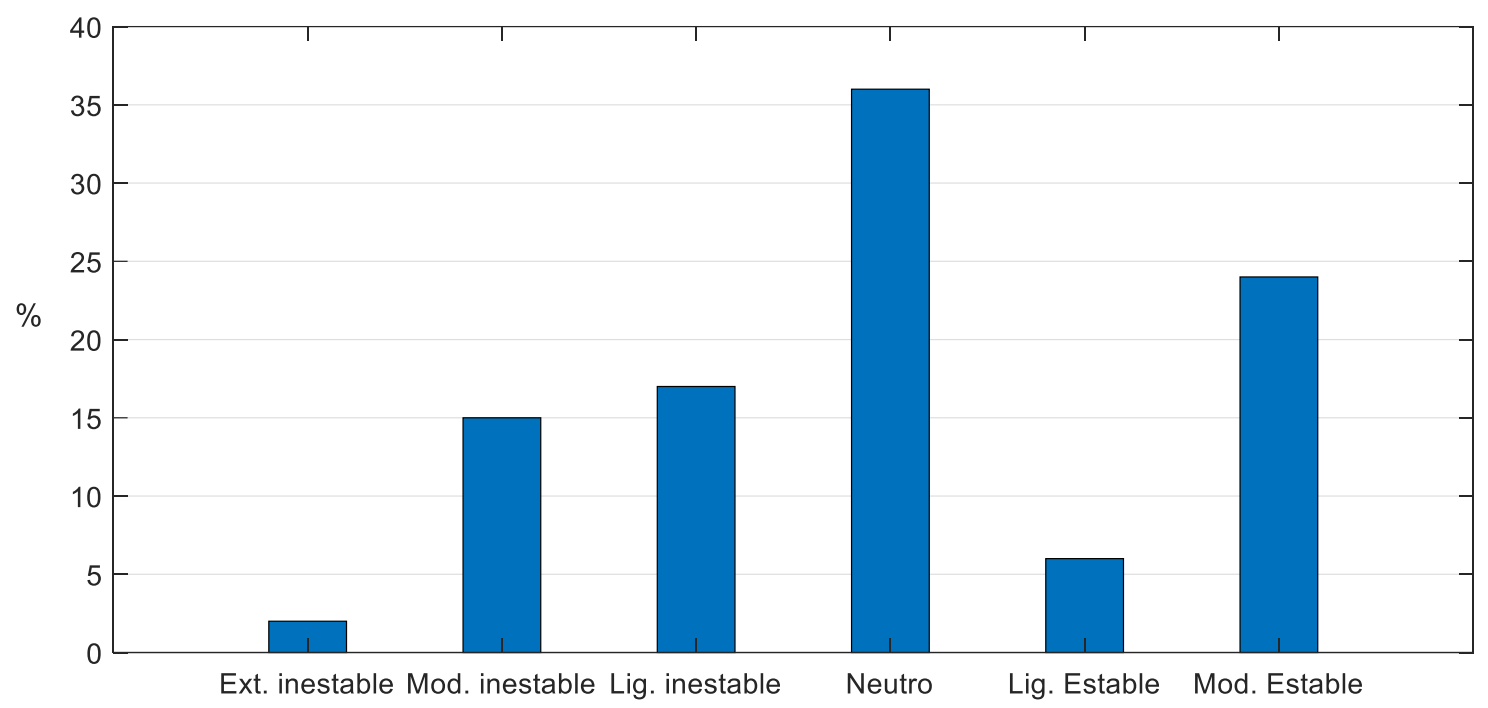

Figura 2. Porcentaje de casos por clase de estabilidad de Pasquill -Turner

Tabla 3. a) Clases de estabilidad por el método del gradiente de temperatura; y b) Índices para ser utilizados en el método del gradiente de temperatura para 10 y $30 \mathrm{~m}$ de altura.

a)

$$
\begin{aligned}
& \text { Velocidad } \\
& \text { del viento }
\end{aligned}
$$$$
\Delta T / \Delta \mathrm{Z}\left({ }^{\circ} \mathrm{K} / 100 \mathrm{~m} \text {, medidos entre } 20 \text { y } 120 \mathrm{~m}\right)
$$

$(\mathrm{m} / \mathrm{s})$ a $10 \mathrm{~m}$

$0-1$

$1-2$

$2-3$

$3-5$

$5-7$

$>7$

\begin{tabular}{c}
$\leq-1.5 \quad-1.4$ \\
\hline A \\
A \\
A \\
B \\
C \\
D
\end{tabular}

b)

Adicionalmente, se utilizó un tercer método (Torres, 2008) para obtener el índice de estabilidad, relacionado con la clase de insolación (radiación neta) y la velocidad del viento, que es una variación del método desarrollado por Panofky y Dutton (1984), el cual se explica en la Tabla 4. 
Tabla 4. a) Cálculos de la clase de estabilidad utilizando la radiación neta y b) la velocidad del viento.

\begin{tabular}{ccc}
\hline a) Radiación solar $\left(\mathbf{w} / \mathbf{m}^{\mathbf{2}}\right)$ & Insolación & Clase de insolación \\
\hline $\mathrm{R}>917.10$ & FUERTE & 4 \\
$567.95<\mathrm{R} \leq 917.10$ & MODERADA & 3 \\
$231.35<\mathrm{R} \leq 567.95$ & DÉBIL & 2 \\
$20<\mathrm{R} \leq 231.35$ & MUY DÉBIL & 1 \\
$\mathrm{R} \leq 20$ & NOCHE & 0
\end{tabular}

\begin{tabular}{cccccc}
\hline $\begin{array}{c}\text { b) Velocidad } \\
\text { del viento }(\mathbf{m} / \mathbf{s})\end{array}$ & $\mathbf{4}$ & $\mathbf{3}$ & $\mathbf{2}$ & $\mathbf{1}$ & $\mathbf{0}$ \\
\hline $\mathrm{V} \leq 0.5$ & 1 & 1 & 2 & 3 & 7 \\
& & & & & \\
$0.5<\mathrm{V} \leq 1.5$ & 1 & 2 & 2 & 3 & 6 \\
$1.5<\mathrm{V} \leq 2.5$ & 1 & 2 & 3 & 4 & 6 \\
$2.5<\mathrm{V} \leq 3.5$ & 2 & 2 & 3 & 4 & 5 \\
$3.5<\mathrm{V} \leq 4.5$ & 2 & 3 & 3 & 4 & 5 \\
$4.5<\mathrm{V} \leq 5.5$ & 3 & 3 & 4 & 4 & 4 \\
$\mathrm{~V}>5.5$ & 3 & 4 & 4 & 4 & 4 \\
\hline
\end{tabular}

Fuente: Agustín Torres Jerez. "Aplicación Práctica del Modelo de Dispersión de Contaminantes Atmosféricos".

\section{Resultados.}

Utilizando los tres métodos se calcularon los índices de estabilidad en la Estación Meteorológica UNL, los cuales presentaron diferencias, en especial el método 2 (M2) con un bajo porcentaje de casos de la clase de estabilidad neutral (Fig. 3), pero en el contexto de los tres métodos el predominante es el neutro.

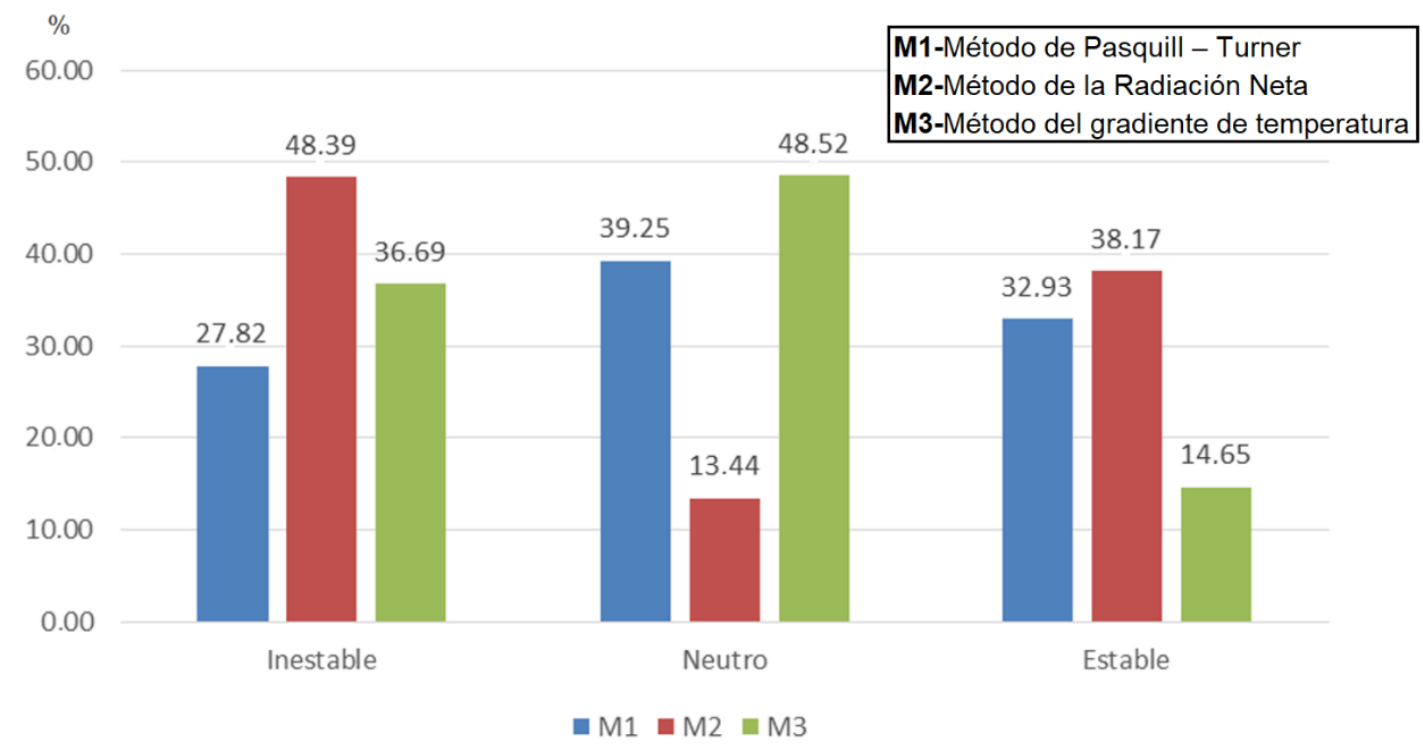

Figura 3. Comparación de resultados de cálculo de los índices de estabilidad por los tres métodos para la Estación Meteorológica UNL. 
Utilizando el software Silverfrost Fortran FTN95, se recalculó la desviación estándar del viento horizontal por horas, utilizando los datos de dirección del viento cada $30 \mathrm{~s}$ de la estación a dos niveles. El procesamiento de la estabilidad atmosférica, mediante gradiente de temperatura, se muestra en la Tabla 5.

Tabla 5. Procesamiento de variables meteorológicas a 10 y $30 \mathrm{~m}$ para calcular la estabilidad atmosférica $\lambda$

\begin{tabular}{ccccccccccc}
\hline $\mathbf{t}_{\mathbf{1}}$ & $\mathbf{t}_{\mathbf{2}}$ & $\boldsymbol{\Delta} \mathbf{t}$ & $\mathbf{C o e f}$ & $\boldsymbol{\lambda}_{\mathbf{t} \mathbf{1}}$ & $\boldsymbol{\lambda}_{\mathbf{t} \mathbf{2}}$ & $\boldsymbol{\lambda}_{\mathbf{t} \mathbf{3}}$ & $\boldsymbol{\lambda}_{\mathbf{t} \mathbf{4}}$ & $\boldsymbol{\lambda}_{\mathbf{t} \mathbf{5}}$ & & \\
& & & $\mathbf{- 0 . 2 8 / -}$ & $\mathbf{- 0 . 2 2 / -}$ & $\mathbf{- 0 . 1 6 / -}$ & - & & & $\boldsymbol{\lambda}_{\mathbf{t} \mathbf{}}$ & $\mathbf{l}_{\mathbf{t 1} \mathbf{u} \mathbf{1 0}}$ \\
${ }^{\circ} \mathbf{C}$ & ${ }^{\circ} \mathbf{C}$ & $\mathbf{< - 0 . 3}$ & $\mathbf{0 . 2 4}$ & $\mathbf{0 . 1 8}$ & $\mathbf{0 . 1 4}$ & $\mathbf{0 . 1 2 / 0 . 0}$ & $\mathbf{0 . 0 2 / 0 . 4}$ & $>\mathbf{0 . 4}$ & & \\
\hline 16.90 & 16.40 & -0.50 & -0.50 & 1.00 & FALSO & FALSO & FALSO & FALSO & FALSO & 1 \\
18.70 & 18.20 & -0.50 & -0.50 & 1.00 & FALSO & FALSO & FALSO & FALSO & FALSO & 1 \\
17.90 & 17.60 & -0.30 & -0.30 & 1.00 & FALSO & FALSO & FALSO & FALSO & FALSO & 1 \\
16.60 & 16.50 & -0.10 & -0.10 & FALSO & 4.00 & FALSO & FALSO & FALSO & FALSO & 4 \\
16.60 & 16.50 & -0.10 & -0.10 & FALSO & 4.00 & FALSO & FALSO & FALSO & FALSO & 4 \\
16.40 & 16.30 & -0.10 & -0.10 & FALSO & FALSO & 4.00 & FALSO & FALSO & FALSO & 4 \\
16.20 & 16.10 & -0.10 & -0.10 & FALSO & FALSO & 4.00 & FALSO & FALSO & FALSO & 4 \\
16.20 & 16.10 & -0.10 & -0.10 & FALSO & FALSO & 4.00 & FALSO & FALSO & FALSO & 4 \\
15.50 & 15.40 & -0.10 & -0.10 & FALSO & FALSO & 4.00 & FALSO & FALSO & FALSO & 4 \\
14.70 & 14.80 & 0.10 & 0.10 & FALSO & FALSO & 5.00 & FALSO & FALSO & FALSO & 5 \\
15.60 & 15.50 & -0.10 & -0.10 & FALSO & FALSO & 4.00 & FALSO & FALSO & FALSO & 4 \\
15.30 & 15.30 & 0.00 & 0.00 & FALSO & FALSO & 4.00 & FALSO & FALSO & FALSO & 4 \\
14.90 & 14.90 & 0.00 & 0.00 & FALSO & FALSO & 4.00 & FALSO & FALSO & FALSO & 4 \\
14.80 & 14.70 & -0.10 & -0.10 & FALSO & 4.00 & FALSO & FALSO & FALSO & FALSO & 4 \\
15.00 & 14.90 & -0.10 & -0.10 & FALSO & 4.00 & FALSO & FALSO & FALSO & FALSO & 4 \\
15.00 & 14.90 & -0.10 & -0.10 & FALSO & FALSO & FALSO & 4.00 & FALSO & FALSO & 4 \\
15.10 & 15.00 & -0.10 & -0.10 & FALSO & FALSO & FALSO & 4.0O & FALSO & FALSO & 4 \\
\hline
\end{tabular}

Ahora bien, con la determinación de la estabilidad atmosférica es permisible calcular el valor de la estratificación atmosférica, con el fin de establecer la concentración máxima de contaminantes utilizando la ecuación de Berlyand.

El modelo utilizado es una modificación a la ecuación de la concentración máxima $\left(C_{m}\right)$ propuesta por Berlyand (1975)

$$
C_{m}=\frac{A M F_{m n}}{H^{2} \sqrt[3]{V \Delta T}}
$$

donde:

$A$, Coeficiente, calculado para condiciones normales de intercambio vertical y horizontal $M$, Cantidad de materia expulsad $\left(\mathrm{g} . \mathrm{s}^{-1}\right)$.

$F$, Coeficiente adimensional para las condiciones de salida de la mezcla gas-aire en el punto de emisión. Para expulsiones gaseosas y aerosoles, $F=1$.

$H$, Altura de la fuente $(\mathrm{m})$.

$V$, Volumen de la mezcla gas-aire $\left(\mathrm{m} 3 \mathrm{~s}^{-1}\right)$. 
$\Delta T$, Diferencia de temperaturas entre e aire y la mezcla gaseosa.

$m n$, coeficientes adimensionales.

La distancia a la cual ocurre la concentración máxima $\left(x_{m}\right)$ se calcula considerando la altura de la fuente $(H)$, el coeficiente adimensional de filtrado $(F)$, y un parámetro $(d)$ que depende de la llamada velocidad peligrosa del viento $\left(U_{m}\right)$, la cual es función del volumen de la mezcla gas-aire y de $\Delta T$.

Debido a que en el presente estudio se pretende calcular el coeficiente $A$ para las condiciones de la Ciudad de Loja, y al no contar con los datos primarios ni secundarios, se utilizó la ecuación de concentración máxima propuesta por el Dr. Berlyand, la cual requiere un valor de $A$ adecuado a las condiciones geográficas y de turbulencia de la zona donde se vaya a aplicar. Para ello se utilizó la ecuación modificada de Berlyand (1994), siendo una más sencilla basada en las características del intercambio vertical $(\delta Z)$ y horizontal $(\delta Y)$, que es la usada en el presente trabajo y que expresa:

$$
A=0.3 \frac{\delta Z}{\delta Y}
$$

Donde $\delta Z$ viene dado por los valores del coeficiente de intercambio vertical $k_{z} y$ velocidad del viento $u$ a la altura $z_{1}=10 \mathrm{~m} ; \delta Y$ es la dispersión de las fluctuaciones de la dirección del viento para un intervalo de tiempo entre 20 - 30 minutos, para el cual las concentraciones son estimadas. Finalmente, para calcular los valores de $\delta Z, \delta Y$ se puede utilizar las ecuaciones formuladas por Briggs, según Ulriksen (2005), que se los detalla en la Tabla 6:

Tabla 6. Fórmulas recomendadas por Briggs según Ulriksen (2005) para $\delta Y(\phi)$ y $\delta Z(h)$

\begin{tabular}{ccc}
\hline Categorías de Pasquill & $\delta Y(\mathrm{~m})$ & $\delta Z(\mathrm{~m})$ \\
\hline & CONDICIONES RURALES & \\
\hline A & $0.22 \phi(1+0.0001 \phi)-0.5$ & $0.20 h$ \\
B & $0.16 \phi(1+0.0001 \phi)-0.5$ & $0.12 \mathrm{~h}$ \\
C & $0.011 \phi(1+0.0001 \phi)-0.5$ & $0.08 h(1+0.0002 h)-0.5$ \\
D & $0.08 \phi(1+0.0001 \phi)-0.5$ & $0.06 h(1+0.0015 h)-0.5$ \\
E & $0.06 \phi(1+0.0001 \phi)-0.5$ & $0.03 h(1+0.0003 h)-1$ \\
F & $0.04 \phi(1+0.0001 \phi)-0.5$ & $0.016 h(1+0.0003 h)-1$ \\
& CONDICIONES URBANAS & \\
A-B & $0.32 \phi(1+0.0004 \phi)-0.5$ & $0.24 h(1+0.001 h)-0.5$ \\
C & $0.22 \phi(1+0.0004 \phi)-0.5$ & $0.20 h$ \\
D & $0.16 \phi(1+0.0004 \phi)-0.5$ & $0.14 h(1+0.0003 h)-0.5$ \\
E-F & $0.11 \phi(1+0.0004 \phi)-0.5$ & $0.08 h(1+0.00015 h)-0.5$
\end{tabular}

El valor de $\phi$ puede ser determinado de la relación de la desviación estándar del viento con respecto al valor medio de los valores medidos cada $30 s$ (longitud de la cuerda). Basándose en análisis de escala, bajo condiciones neutras, la altura de la capa límite suele calcularse a partir de la expresión presentada por Holtslag A.A.M. y van Ulden A.P. (1983): 


$$
h \approx 125 u
$$

Donde $u$ es la velocidad del viento a la altura de $10 \mathrm{~m}$. El límite superior de la capa superficial se define como la altura en la que $\frac{z}{h}=0.1$, siendo $h$ la altura de la capa límite. La longitud de Monin- Obukhov se calculó utilizando la ecuación $L^{2}=K^{2} Z^{2}$, donde $K$ es la constante de Von Karman (0.37) y $Z$ es el límite superior de la capa superficial.

Para el exponente de perfil de viento en terrenos no complejos, Turtós propone una ecuación hasta una altura de $200 \mathrm{~m}$ sobre el nivel del terreno, considerando que el perfil de viento está razonablemente bien representado por la ley de potencia (Turtós y otros, 2004):

$$
U_{z}=U_{r}\left(\frac{Z}{Z_{r}}\right)^{p}
$$

donde $U_{r}$ es la velocidad escalar media de viento a la altura de referencia $Z_{r}$, típicamente 10 metros.

Para el caso de la Ciudad de Loja, la cual se encuentra en un valle entre montañas, con zonas de grandes pendientes, se calculó una longitud de rugosidad orográfica para determinar si la zona en la cual está enclavada la ciudad se puede considerar relativamente plana. Esto se realizó confeccionando el Modelo Numérico de Altitud, utilizando los datos del Shuttle Radar Topographic Model (SRTM) con resolución de 90 m, al cual posteriormente se le calculó la desviación estándar para modelar la superficie de rugosidad orográfica (Fig. 4), donde se puede observar que la ciudad se encuentra en una zona con valores casi constantes y aproximadamente igual a 0.1 .

El exponente $p$ varía usualmente desde 0.1 en una tarde soleada hasta 0.6 durante noches despejadas. Mientras mayor sea el valor de $p$, mayor será el gradiente vertical de la velocidad del viento. Como esta ley de potencia es una aproximación del perfil medio de velocidad del viento, los perfiles reales se desvían de esta relación. Los valores de $p$, específicos para cada sitio, pueden determinarse con los datos de vientos en dos niveles, resolviendo la ecuación (Turtós y otros, 2004):

$$
p=\frac{\ln (U)-\ln \left(U_{r}\right)}{\ln (Z)-\ln \left(Z_{r}\right)}
$$

En nuestro caso se utilizaron los datos de viento a las alturas de $10 \mathrm{~m}$ y $30 \mathrm{~m}$. El gradiente vertical y el gradiente potencial de temperatura son usados ampliamente en la modelación de la dispersión de los contaminantes en la atmósfera para clasificar la estabilidad en la capa superficial, utilizando algoritmos de parametrización de datos de superfice como la altura de la capa de mezcla y en las ecuaciones de elevación del penacho para condiciones estables (las de menor porcentaje de ocurrencia en nuestro caso). Estos gradientes se obtienen, internacionalmente, de los sondeos diarios (Turtós y otros, 2004). En Ecuador estos sondeos solamente se realizan en tres lugares, específicamente en Guayaquil (5 m 
de elevacion en costa), isla San Cristóba (6 m de elevación en Galápagos) y en la estación Nuevo Rocafuerte a una altura de 264 msnm.

La longitud de rugosidad puede ser calculada a partir de las mediciones de los perfiles de viento. De hecho, en caso de turbulencia puramente mecánica (por ejemplo, con vientos fuertes), la velocidad del viento promedio u muestra un perfil de viento logarítmico para Z > Z0, el cual está dado por (Panofsky y Dutton, 1984).

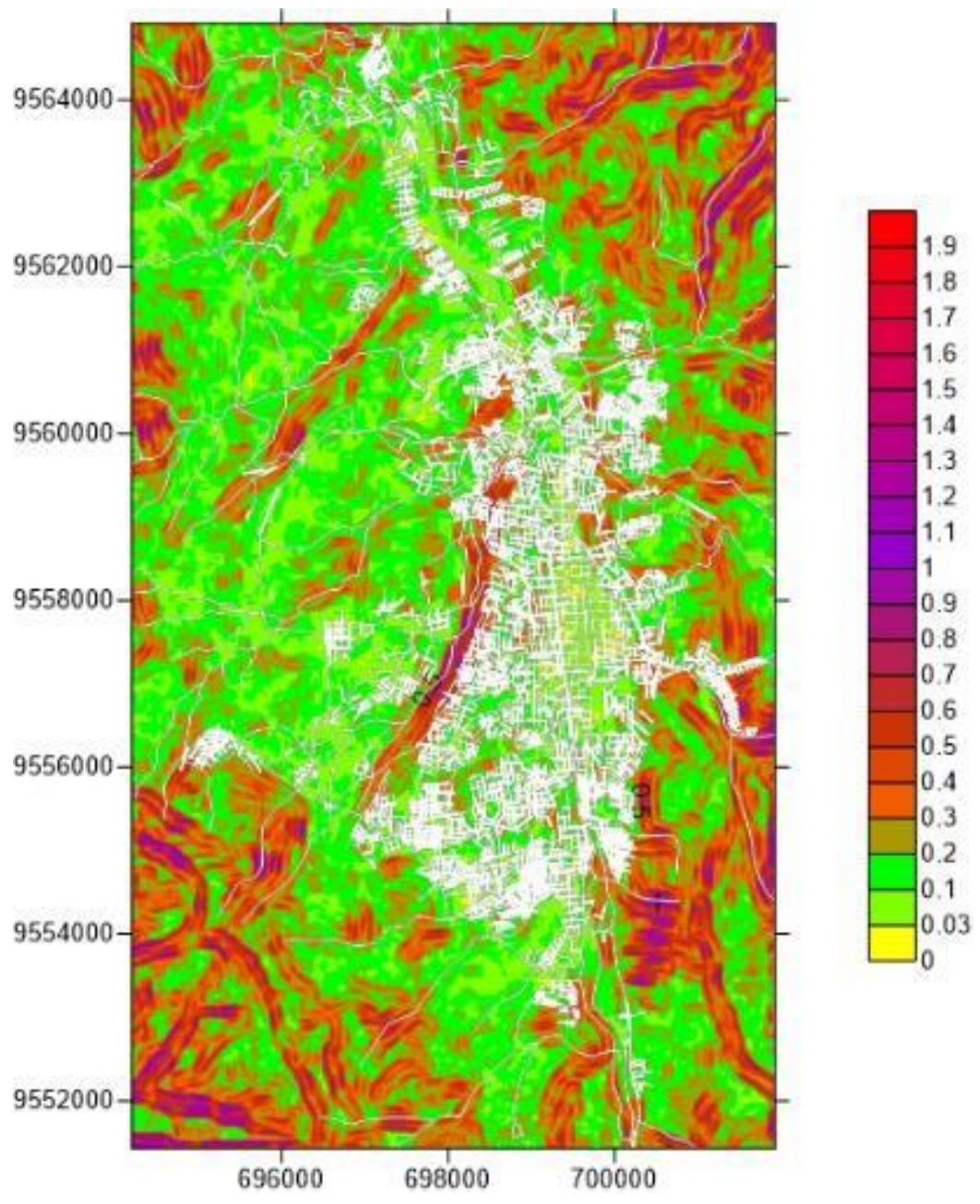

Figura 4. Superficie de rugosidad orográfica para la ciudad de Loja.

El procesamiento de los cálculos de las Eq. 1 - Eq. 5 se los realizó en una hoja Excel, cuyos resultados importantes se muestran en la Tabla 7.

Tabla 7. Resultados de los cálculos para la dispersión, la estabilidad atmosférica y el coeficiente

$\boldsymbol{A}$ de la ecuación de Berlyand.

\begin{tabular}{cccc}
\hline $\boldsymbol{\delta Z}$ & $\boldsymbol{\lambda}$ & $\boldsymbol{\delta} \boldsymbol{Y}$ & $\boldsymbol{A}$ \\
\hline 46 & 2 & 0,22 & 83
\end{tabular}

Utilizando la ecuación propuesta por Berlyand (1994), para cada hora y cada mes, se calculó el coeficiente $A$. Previo a utilizar los resultados de la estación meteorológica a dos 
niveles $(10$ y $30 \mathrm{~m})$ se utilizó la información del trabajo sobre estabilidad vertical de la atmósfera en la provincia de Loja (Álvarez, Maldonado y Montaño, 2015) procesando y calculando los valores para la Ciudad de Loja, obteniendo una estabilidad neutra.

Como resultado del procesamiento de las observaciones de temperatura del aire, y de dirección y fuerza del viento a los niveles de 10 y $30 \mathrm{~m}$ obtenidos de la estación meteorológica automática que se ubicó en los terrenos de la Universidad Nacional de Loja, durante los meses de enero a marzo de 2015, se obtuvieron, como promedios, los resultados que se muestran en la Tabla 7 , en la cual se pueden observar los parámetros de dispersión

$\delta Y(\phi)$ y $\delta Z(h)$, la clase de estabilidad, así como el valor del coeficiente $A$, para las condiciones de la ciudad de Loja (Montaño, T. 2015).

\section{Conclusiones.}

- En este estudio se determinó el valor del coeficiente de estratificación horizontal y vertical (denominado coeficiente $A$ o parámetro $A$ ) de la ecuación de Berlyand para el cálculo de la concentración máxima de gases a partir de fuentes fijas puntuales, el cual resultó con un valor de 83. El valor promedio calculado del parámetro $A$ se corresponde con lo planteado por Berlyand (1975) como perteneciente a zonas sin gran turbulencia en las zonas centrales de la antigua URSS (valor 80), lo cual se cumple en la ciudad de Loja, en la cual, al contar con de nubes de tipo convectivo, no pasan de cúmulos promedios en la mayoría de los casos, no reportándose tormentas eléctricas con frecuencia. Adicionalmente, en el período de mediciones el promedio de la clase de estabilidad corresponde a la categoría neutra.

- Adicionalmente a las conclusiones de este trabajo de investigación, los autores recomiendan realizar la modelación a partir de los datos técnicos medidos en las distintas fuentes, y considerar las matrices de viento (por valores de velocidad dirección) como datos de control para el cálculo de la concentración máxima utilizando el parámetro $A$ calculado para la ciudad de Loja.

\section{Agradecimiento.}

Los autores agradecen el financiamiento de la Universidad Nacional de Loja a través del proyecto de investigación 28-DI-FEIRNNR-2019 'Caracterización de la potencialidad de la energía solar y eólica en la Región Sur del Ecuador.'

\section{Referencias bibliográficas.}

Agencia Nacional de Transito 2014. Estadística de matriculación 2013 (Consultado 15 julio, 2014). 
Arya, S Pal, 2002: A Review of the Theoretical Bases of Short-Range Atmospheric Dispersion and Air Quality Models. Proc. Indian Natn Sci Acad, 69, A, No 6, November 2003, pp 709-724.

Álvarez, O.H., Maldonado, J. y Montaño, T. (2015): Estabilidad vertical de la atmósfera en la provincia de Loja, Ecuador (inédito).

Berlyand, M.E. (1975): Problemas actuales de la difusión atmosférica y la contaminación de la atmósfera. Gidrometeoizdat, Leningrado (en ruso).

Berlyand, M.E. (1994) Actual problems of development of air pollution modelling and its influence on the environment. Main Geophysical Observatory, St. Petersburg. Russia, (en ruso)

CEPAL (2008):“Anuario estadístico de América Latina y el Caribe”, Chile.

Delgado, M., D. Sánchez y S. Zapata: "Sistema de Soporte de Decisiones: Aplicación a la Gestión de la Contaminación en la ciudad de Santiago de Chile”. XII Congreso Español Sobre Tecnologías y Lógica Fuzzy. Universidad de Granada Universidad de Granada. U. Tecnológica Metropolitana.

GEO Loja 2006, 2006: Perspectivas del Medio Ambiente Urbano. ISBN 978-9942-01460-3. Ecuador $192 \mathrm{pp}$.

Holtslag A.A.M. y van Ulden A.P. (1983): A simple scheme for daytime surface fluxes from routine weather data. J. of Climatic and Applied Meteorology 22, 517-529.

Holzworth, G. C. (1974): "Climatological Aspects of the Composition and Pollution of the Atmosphere". TECHNICAL NOTE No. 139. WMO - No. 393. ISBN 92-6310393-3.

INEC 2010: Población a nivel Parroquias. www.location-world.com (noviembre, 2014)

Montaño, T. (2015). Metodología para evaluar los gases contaminantes que emiten las fuentes puntuales que causan impactos ambientales en la ciudad de Loja ubicada al Sur del Ecuador. Tesis de doctorado en Ciencias Ambientales. Universidad Nacional de Piura. Perú.

Moragues, J.A. (2015): Clases de Estabilidad. Capas de Mezcla. Disponible en: http://www.ceiucaweb.com.ar/documentos/2-ambiental/3er-anio-1ercuatri/meteorologia/apunte /capa\%20de\%20mezcla.pdf

Neiburger, M , (1969), The role of meteorology in the study and control of air pollution Bull. Am. Meteorol. Soc. , 50, 957-965

OMS (2011) Calidad del Aire y Salud. Disponible en:

http://www.who.int/mediacentre/factsheets/fs313/es/ (Consultado Abril 22, 2014). 
Panofsky, H.A. y Dutton, J.A. (1984): Atmospheric Turbulence: Models and Methods for Engineering Applications. Wiley \& Sons, New York, 397 pp.

PNUMA, 2008: Anuario 2008 del PNUMA. ISBN: 978-92-807-2880-4. $\mathrm{UNEP} / \mathrm{GCSS} / \mathrm{X} / \mathrm{INF} / 2$.

PNUMA, I. Municipio de Loja, Naturaleza y Cultura Internacional (2008):"Perspectivas del Medio Ambiente Urbano; Geo Loja". Disponible en: http://www.naturalezaycultura.org/ (Consultado Abril 4, 2014).

S Pal Arya, 2002: A Review of the Theoretical Bases of Short-Range Atmospheric Dispersion and Air Quality Models. Proc. Indian Natn Sci Acad, 69, A, No 6, November 2003, pp 709-724.

Torres, A. EOI. Aplicación práctica del modelo de dispersión de contaminantes atmosféricos - ISCST3. Máster en Ingeniería y Gestión Medioambiental.20072008 .

Turtós, L., y otros (2004): Salida 9 (2/2004): Propuesta de Guía para realizar los estudios de dispersión local de contaminantes gaseosos y partículas. Externalidades Ambientales Atmosféricas de la Generación Eléctrica. Proyecto programa ramal de desarrollo energético sostenible, La Habana, Cuba. Junio 2004.

Ulriksen, P. (2005): Apuntes, Modelos de Dispersión de Contaminantes. Universidad de Chile, Facultad de Ciencias Físicas y Matemáticas. Escuela de Postgrado, Diploma en Contaminación Atmosférica.

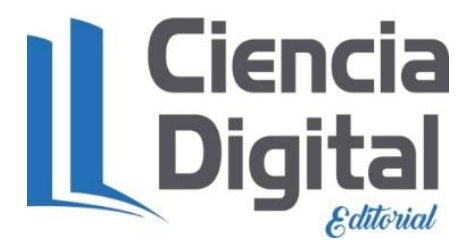




\section{PARA CITAR EL ARTÍCULO INDEXADO.}

Solano-Jiménez, J., Montaño-Peralta, T., Álvarez-Hernández, O., León-Tapia, M., Torres-R, W., \& Montaño-Ramón, T. (2021). Determinación del coeficiente de estratificación horizontal y vertical de la ecuación modificada de Berlyand para fuentes fijas en la ciudad de Loja-Ecuador. ConcienciaDigital, 4(1.2), 103-118. https://doi.org/10.33262/concienciadigital.v4i1.2.1582

\section{¿Ciencia}

El artículo que se publica es de exclusiva responsabilidad de los autores y no necesariamente reflejan el pensamiento de la Revista Ciencia Digital.

El artículo queda en propiedad de la revista y, por tanto, su publicación parcial y/o total en otro medio tiene que ser autorizado por el director de la Revista Ciencia Digital.
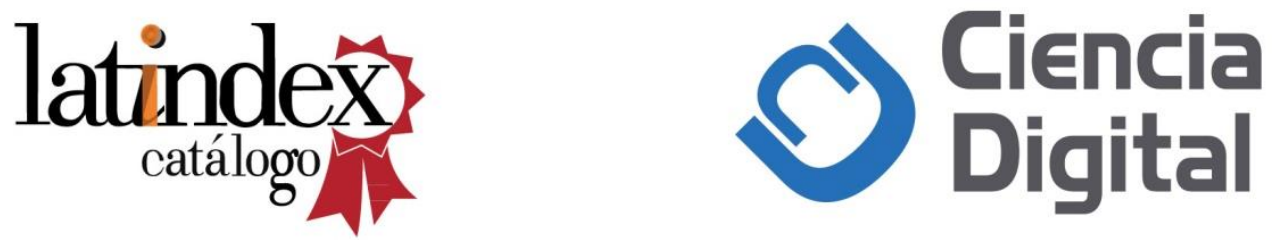